\title{
Clinical Reasoning: Myokymia, Dysautonomia, and Uveitis Researching a Common Denominator
}

Mikhail $\mathrm{M}^{1}$, Maurice $\mathrm{C}^{2^{*}}$

${ }^{1}$ Faculty of Medicine, University of Toronto, Toronto, Canada

${ }^{2}$ Pencer Brain Tumor Centre, Princess Margaret Hospital Cancer Centre, Toronto, Canada

Corresponding Author: Catherine Maurice, MD, FRCPC

Address: Neuro-Oncologist, Princess Margaret Cancer Centre, 610 University Avenue, Office: 18-717, Toronto, ON, Canada, M5G 2M9; Office Phone: 416-946-2277; Office Fax: 416-946-2284; Email: Catherine.Maurice@uhn.ca Received date: 29 November 2019; Accepted date: 19 December 2019; Published date: 2 January 2020

Citation: Mikhail M, Maurice C. Clinical Reasoning: Myokymia, Dysautonomia, and Uveitis Researching a Common Denominator. Asp Biomed Clin Case Rep. 2020 Jan 2;3(1):22-24.

Copyright (c) 2020 Mikhail M, Maurice C. This is an open-access article distributed under the Creative Commons Attribution License, which permits unrestricted use, distribution, and reproduction in any medium provided the original work is properly cited.

\section{Keywords}

Morvan's Syndrome; Caspr2 Antibody; Paraneoplastic Syndrome; Limbic Encephalitis; Dysautonomia; Uveitis; Neuromyotonia; Mycophenolate Mofetil

\author{
Abbreviations \\ EMG: Electromyography; Caspr2: Contactin Associated Protein-Like 2; CT: Computed Tomography; PET: \\ Positron Emission Tomography
}

\section{Section-1}

A 56-year old man, originally from Pakistan, presented with bilateral avascular necrosis of the hips on a background of emphysema, pulmonary fibrosis, coronary artery disease, diabetes type 2 and psoriasis. The cause of the avascular necrosis was unclear, with no recent trauma or steroid use. During his preoperative consultation, he presented dysautonomia requiring an inpatient investigation; the surgery was canceled. He reported a thirtypound weight loss and a three-week history of night sweats and shortness of breath. While hospitalized, this gentleman presented subacute confusion and fluctuation of his sensorium, compatible with limbic encephalitis. Furthermore, he developed diffuse myokymia involving the axial and appendicular musculature, confirmed by EMG.

\section{Questions for consideration:}

1. What is the most probable diagnosis combining subacute limbic encephalitis, dysautonomia, and myokymia?

2. What workup should be advised?

\section{Section-2}

Due to the combination of limbic encephalitis and myokymia, a paraneoplastic work-up was conducted. Caspr2 and Anti-Jo-1 antibodies were documented in the serum. Caspr2 is a brain and peripheral nerve autoantigen associated with voltage-gated potassium junctions that may result in encephalitis, peripheral nerve dysfunction, or a combination of both, known as Morvan's syndrome [1]. Our patient presented this rare autoimmune condition characterized by neuromyotonia, central nervous system involvement 
(confusion, agitation, hallucinations) and autonomic features (hyperhidrosis, tachycardia, dysautonomia) $[2,3]$. The diagnosis of Morvan's syndrome prompted an examination of the underlying antigenic source. Thymoma was ruled-out, and a thorough investigation excluded the possibility of an active neoplastic process [4].

\section{Questions for consideration:}

1. Considering that no cancer was detected, which other concomitant aetiologies should trigger our attention in association with the onset of a paraneoplastic syndrome?

\section{Section-3}

In addition to malignancies, multiple autoimmune conditions, associated with autoantibodies, might be associated with the onset of a paraneoplastic syndrome. Our patient has originally been investigated for arthritis in the context of avascular necrosis. This workup failed to document rheumatoid arthritis. Aside from his diagnosis of pulmonary fibrosis, this gentleman subsequently developed uveitis, responding to steroids. Thus, a rheumatological disorder was highly suspected at this point; explaining the development of various symptoms all linked to autoimmunity in a restricted lapse of time. The presence of anti-Jo antibodies was of unclear significance, the specificity of this test being limited.

\section{Questions for consideration:}

1. What treatment is recommended? What are the potential side effects of this treatment?

Due to the concerning fluctuations in heart rate and blood pressure, the hip replacement surgery was not conducted. Our patient was prescribed mycophenolate mofetil, leading to a complete recovery. Regarding the long-term course, opportunistic infections and the challenges of immunosuppression remained the main concerns. Prednisone was necessary when the uveitis appeared. It was challenging to taper the prednisone; however several symptoms described were in retrospective side effects of the steroids, complicating even more this clinical scenario.

Typically, paraneoplastic syndromes implicate an underlying malignant origin, provoking an immunemediated response. However, in patients presenting anti-voltage-gated potassium channel antibodies, only $47 \%$ are found to have cancer, meaning that in fact, the majority of patients with this condition are cancer-free [5]. On the other hand, this assumption could not be presumed upfront. A thorough follow-up includes PET scans semi-annually, as well as thoracicabdo-pelvic CT scans on a quarterly basis. Furthermore, global systemic conditions should be considered in the context of this patient's uveitis, as there are no ocular manifestations associated with Morvan's syndrome. The presence of anti-Jo and the result of the muscle biopsy suggested concomitant polymyositis. If this patient was indeed individual suffering from multiple concomitant immunemediated conditions, all his symptoms should have responded to immunosuppressive therapy. Since paraneoplastic syndromes are renown to present when the underlying cancer is not yet visible by any imaging modality, the persistent research of neoplasia should continue even if the initial work-up is unremarkable. The evolution will eventually determine the management: if the underlying paraneoplastic syndrome completely resolves without any recurrence, an underlying malignancy is less probable and the investigation can be discontinued.

\section{Financial disclosure}

The authors have nothing to disclose. No funding was received in the context of this work.

\section{Authors Contribution}

Mirriam Mikhail: Conceptualization of the manuscript, Research, Background, Redaction, Reviewing, Editing.

Catherine Maurice: Primary supervisor, Conceptualization of the manuscript, Redaction, Reviewing, Editing, Pictures/Figures.

\section{Disclosures}

The authors have nothing to disclose. 


\section{Funding received in the context of this work} None

\section{References}

[1] van Sonderen A, Schreurs MW, Wirtz PW, Sillevis Smitt PA, Titulaer MJ. From VGKC to LGI1 and Caspr2 encephalitis: The evolution of a disease entity over time. Autoimmun Rev. 2016 Oct;15(10):970-74. [PMID: 27485013]

[2] Barber PA, Anderson NE, Vincent A. Morvan's syndrome associated with voltage-gated $\mathrm{K}+$ channel antibodies. Neurology. 2000 Feb 8;54(3):771-72. [PMID: 10680828]
[3] Liguori R, Vincent A, Clover L, Avoni P, Plazzi G, Cortelli P, Baruzzi A, Carey T, Gambetti P, Lugaresi E, Montagna P. Morvan's syndrome: peripheral and central nervous system and cardiac involvement with antibodies to voltage-gated potassium channels. Brain. 2001 Dec;124(Pt 12):2417-26. [PMID: 11701596]

[4] Lee W, Day TJ, Williams DR. Clinical, laboratory and electrophysiological features of Morvan's fibrillary chorea. J Clin Neurosci. 2013 Sep;20(9):1246-49. [PMID: 23712055]

[5] Darnell RB, Darnell R, Posner JB. Paraneoplastic syndromes. OUP USA; 2011 Aug 22.

Keywords: Morvan's Syndrome; Caspr2 Antibody; Paraneoplastic Syndrome; Limbic Encephalitis; Dysautonomia; Uveitis; Neuromyotonia; Mycophenolate Mofetil 